\title{
Porphyrin-Annulene Hybrid
}

Key words

tellurium

porphyrins

annulenes
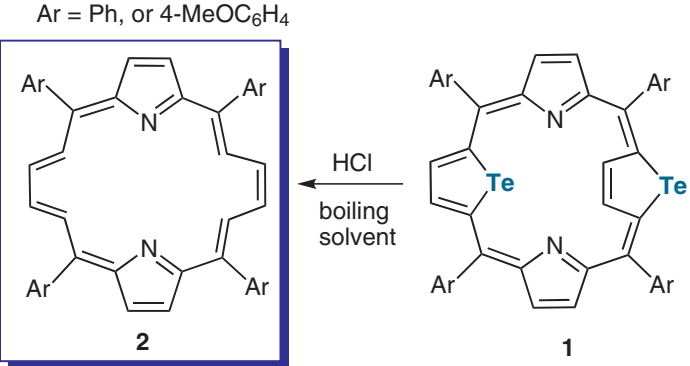

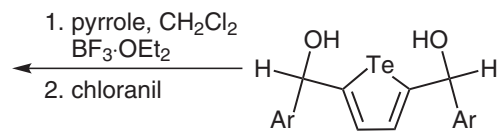

Significance: Porphyrin-annulene hybrid molecule 2 was synthesized via the tellurium-containing macrocyclic intermediate 1. Treatment of $\mathbf{1}$ with $\mathrm{HCl}$ gave $\mathbf{2}$ in a good yield, which is attributed to the facility of tellurium extrusion. The substitution of $\mathrm{HCl}$ by $\mathrm{DCl}$ led to deuterium incorporation at the $\beta$-positions of the tellurophene and the pyrrole moieties.
Comment: The hybrid molecule still possesses porphyrin-like structure and aromaticity, and the flexibility of annulene. The four periphery aryl groups are expected to contribute to the stability of 2. 